\title{
Gamma strength functions and level densities from high-resolution inelastic proton scattering at very forward angles
}

\author{
Sergej Bassauer ${ }^{1, a}$, Peter von Neumann-Cosel ${ }^{1}$, and Atsushi Tamii ${ }^{2}$ \\ 1 Institut für Kernphysik, Technische Universität Darmstadt, 64289 Darmstadt, Germany \\ 2 Research Center for Nuclear Physics, Osaka University, Ibaraki, Osaka 567-0047, Japan
}

\begin{abstract}
Inelastic proton scattering at energies of a few $100 \mathrm{MeV}$ and forward angles including $0^{\circ}$ provides a novel method to measure gamma strength functions (GSF) in nuclei in an energy range of about 5-23 MeV. The experiments provide not only the E1 but also the M1 part of the GSF. The latter is poorly known in heavy nuclei. A case study of ${ }^{208} \mathrm{~Pb}$ indicates that the systematics proposed for the M1-GSF in RIPL-3 needs to be substantially revised. Comparison with gamma decay data (e.g. from the Oslo method) allows to test the generalised Brink-Axel (BA) hypothesis in the energy region of the pygmy dipole resonance (PDR) crucial for the modelling of $(n, \gamma)$ and $(\gamma, n)$ reactions in astrophysical reaction networks. A fluctuation analysis of the high-resolution data also provides a direct measure of level densities in the energy region well above the neutron threshold, where hardly any experimental information is available.
\end{abstract}

\section{Introduction}

Gamma strength functions describe the average gamma decay behaviour of a nucleus as a function of excitation energy. They serve as input in statistical nuclear theory in astrophysics [1], reactor design [2], and waste transmutation [3]. Although all electromagnetic multipoles contribute, GSFs are usually dominated by the E1 component with smaller contributions from M1 strength.

Many applications imply an environment of finite temperature, notably in stellar scenarios [4], and thus reactions on initially excited states become relevant. Their contributions to the reaction rates are usually estimated applying the generalised Brink-Axel hypothesis [5,6] which states that the GSF is independent of the properties of the initial and final states. The validity of the BA hypothesis is also implicitly assumed in the derivation of the GSF from many experimental data based on ground state photoexcitation. Although historically formulated for the IVGDR, where it seems to hold approximately for not too high temperatures [7], the BA hypothesis is nowadays commonly used to calculate the low-energy E1 and M1 strength functions.

Recent work utilising compound nucleus gamma decay with the so-called Oslo method [8] has demonstrated independence of the GSF from excitation energies and spins of initial and final states in accordance with the BA hypothesis once the level densities are sufficiently high to suppress large intensity fluctuations [9]. However, there are a number of experimental results which seem to violate the BA hypothesis in the low-energy region [10-12].

In this proceedings paper recommended parametrisations of the GSF and LD summarised in the RIPL-3 data base [13] are evaluated for the case of ${ }^{208} \mathrm{~Pb}$. In particular,

a e-mail: sbassauer@ikp.tu-darmstadt.de we provide new data for the poorly known M1 part of the GSF. The comparison with the Oslo experiment provides a test of the BA hypothesis. Moreover, since GSF and LD are independently determined, the decomposition of both quantities in the Oslo method, which measures the product of GSF and LD [8], can be verified.

\section{Gamma strength function of ${ }^{208} \mathrm{~Pb}$}

In the experiments discussed here, the GSF for an electric or magnetic transition $X \in\{E, M\}$ with multipolarity $\lambda$ is related to the average photoabsorption cross section $\left\langle\sigma_{a b s}^{X \lambda}\right\rangle$

$$
f^{X \lambda}(E, J)=\frac{2 J_{0}+1}{(\pi \hbar c)^{2}(2 J+1)} \frac{\left\langle\sigma_{a b s}^{X \lambda}\right\rangle}{E^{2 \lambda-1}},
$$

where $E$ denotes the gamma energy and $J, J_{0}$ the spins of excited and ground state, respectively [13]. In practise, only E1 and M1 provide sizeable contributions to the total GSF. In the following, we discuss the derivation of the GSF for these components from the experimental data and compare to parametrisations recommended in RIPL-3.

\subsection{E1 contribution}

The E1 contribution of the GSF in ${ }^{208} \mathrm{~Pb}$ was determined using polarised inelastic proton scattering data obtained at the Research Center for Nuclear Physics in Osaka, Japan with a beam energy of $295 \mathrm{MeV}$ in an excitation energy region from 5 to $23 \mathrm{MeV}[14,15]$. In Ref. [15], the $B(\mathrm{E} 1)$ strength was extracted by means of the multipole decomposition analysis (MDA) in the energy region from 4.8 to $9 \mathrm{MeV}$. The $B(\mathrm{E} 1)$ strength provided in Table I of Ref. [15] was used to determine the E1 part of the GSF. In the IVGDR region, the E1-GSF was determined from 


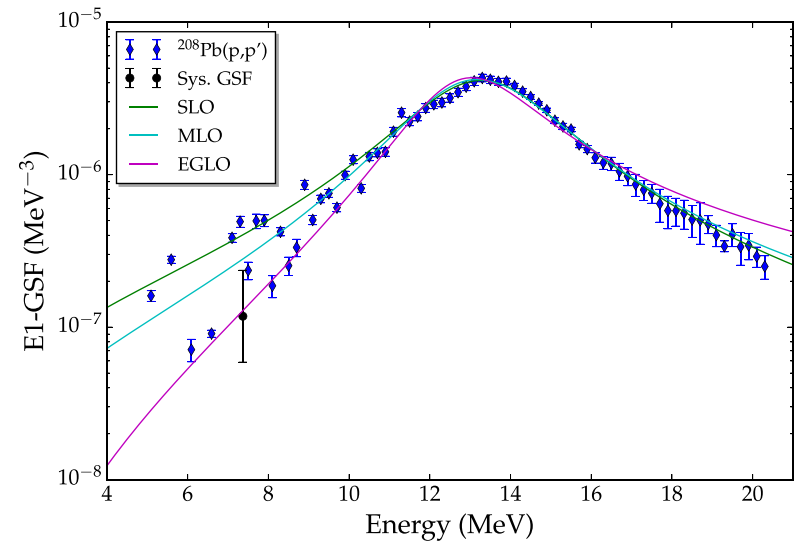

Figure 1. E1-GSF of ${ }^{208} \mathrm{~Pb}$ deduced from the $\left(p, p^{\prime}\right)$ data $[14,15]$ (blue diamonds) in comparison with the SLO (green line), MLO (cyan line) and EGLO (magenta line) models explained in the text. The black circle shows the prediction from experimental systematics at the neutron threshold [17].

photoabsorption cross sections extracted from the $\left(p, p^{\prime}\right)$ data by means of the virtual photon method [14].

The experimental E1-GSF is compared with three widely used models. The simplest model is the standard Lorentzian (SLO) model. This model consists of a single Lorentzian function where the parameters are determined from experimental data [13].

A more sophisticated model is the enhanced generalised Lorentzian (EGLO) model. The EGLO consists of two terms [16], a Lorentzian with an energy- and temperature-dependent width and a term describing the shape of the low-energy part of the GSF. The temperature dependence is estimated within Fermi liquid theory.

The third model used here is the modified Lorentzian model (MLO). In contrast to SLO and EGLO, which are both parametrisations of experimental data, the MLO is based on general relations between the GSF and the imaginary part of the nuclear response function. The MLO is an energy- and temperature-dependent Lorentzian with a scaling factor, which accounts for the enhancement of the GSF with increasing temperature.

The E1-GSF determined from $\left(p, p^{\prime}\right)$ data (blue diamonds) in Fig. 1 is compared to model predictions shown as green (SLO), magenta (EGLO) and cyan (MLO) curves. In the region around the maximum of the IVGDR all models provide a good description. However, the EGLO overestimates the experimental data at higher energies. The low-energy tail of the IVGDR exhibits strong fluctuations which complicate the comparison with smooth strength functions. Between 7 and $8.5 \mathrm{MeV}$ a resonancelike structure dominates the GSF identified as the PDR in ${ }^{208} \mathrm{~Pb}$ [15]. This low-energy resonance is not included in the models. Finally, the GSF value expected at neutron threshold ( $S_{n}=7.37 \mathrm{MeV}$ ) from experimental systematics of neutron capture cross sections (black circle) is almost an order of magnitude smaller than the experimental strengths in the PDR. However, this may be an artefact of the unusually low level density in the doubly magic nucleus ${ }^{208} \mathrm{~Pb}$ with corresponding strong fluctuations of individual strengths at energies close to the neutron threshold (note that the GSF values correspond to energy bins rather

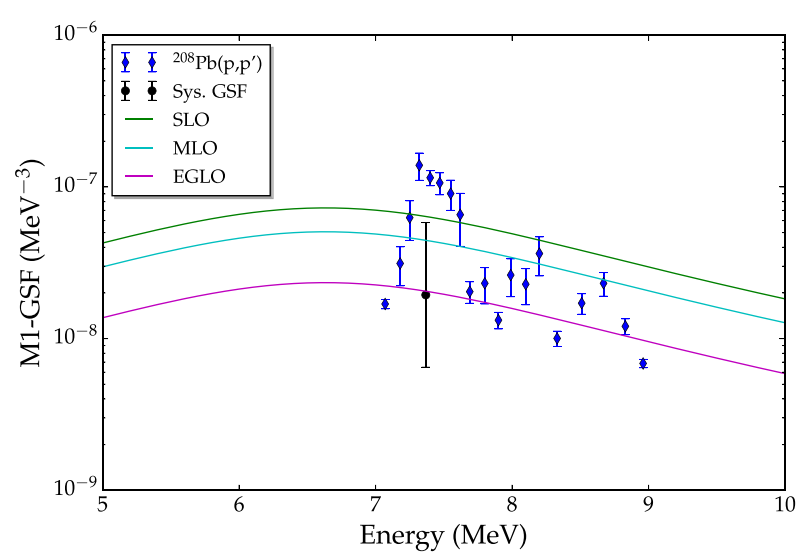

Figure 2. Same as Fig. 1 but for the M1 component of the GSF.

than to individual transitions for excitation energies above $7 \mathrm{MeV}$ (cf. Table I in Ref. [15])).

\subsection{M1 contribution}

M1 cross sections are provided in Table I of Ref. [15]. These are concentrated between 7 and $9 \mathrm{MeV}$ and represent the spinflip M1 resonance [18]. We note that an additional M1 transition to a $1^{+}$state at $5.844 \mathrm{MeV}$ is known (see Ref. [19] and references therein) but omitted here because it is of dominant isoscalar nature [20]. Recently a method utilising isospin symmetry has been presented to relate the cross sections to those of Gamow-Teller excitations studied with the $(p, n)$ reaction and extract the spin-M1 matrix elements [21]. Assuming dominance of the spin-isovector part of the electromagnetic M1 operator reduced $B(\mathrm{M} 1)$ transition strengths can be extracted.

Figure 2 displays the corresponding M1-GSF in comparison with SLO, EGLO and MLO model predictions for ${ }^{208} \mathrm{~Pb}$. The M1-GSF model results are derived from the E1 models discussed above in the following way [13]

$$
f^{M 1}(E)=\frac{f^{E 1}\left(S_{n}\right)}{\mathcal{R}} \frac{\Phi^{M 1}(E)}{\Phi^{M 1}\left(S_{n}\right)}
$$

and

$$
\mathcal{R}=\frac{f^{E 1}\left(S_{n}\right)}{f^{M 1}\left(S_{n}\right)}=0.0588 A^{0.878},
$$

where $\Phi^{M 1}(E)$ is an SLO parametrisation of the spinflip M1 resonance. The ratio $\mathcal{R}$ is valid for nuclei with $S_{n} \approx$ $7 \mathrm{MeV}$. Thus, it should be a good approximation for ${ }^{208} \mathrm{~Pb}$.

The comparison in Fig. 2 indicates that the theoretical GSF values near maximum are of magnitudes roughly comparable to the data. However, the assumed resonance properties represent a poor approximation of the data. The theoretical maxima are about $500 \mathrm{keV}$ too low and the experimental width is grossly overestimated. As a result, the predicted total strengths of the spinflip M1 resonance exceed the experimental value $\sum B(\mathrm{M} 1)=20.5(13) \mu_{N}^{2}$ [21] by factors ranging from two (EGLO) to five (SLO).

\subsection{Total GSF and comparison with Oslo data}

The total GSF summing all contributions is displayed in Fig. 3 (blue diamonds) and compared to data derived with the Oslo method from a ${ }^{208} \mathrm{~Pb}\left({ }^{3} \mathrm{He},{ }^{3} \mathrm{He}^{\prime} \gamma\right)$ experiment 


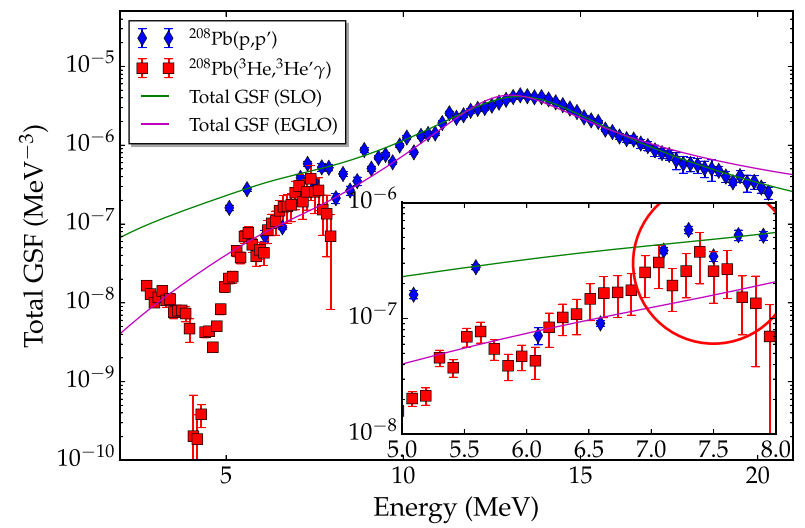

Figure 3. Total GSF of ${ }^{208} \mathrm{~Pb}$ from the $\left(p, p^{\prime}\right)$ data $[14,15]$ in comparison to the reanalysed [23] results from the Oslo experiment [22]. The inlet shows an expanded view of the lowenergy region 5-8 $\mathrm{MeV}$.

(red squares) [22]. The data set has been reanalysed recently [23]. The main changes are new, updated response functions for the CACTUS detector array and an improved error estimate in the simultaneous extraction of the LD and GSF from the primary $\gamma$-ray spectra.

There are overlapping results from both experiments in the energy region between 5 and $8 \mathrm{MeV}$ (see inlet of Fig. 3). The GSF derived from the $\left(p, p^{\prime}\right)$ data is systematically higher in the PDR region although they seem still compatible within error bars in the peak region around the neutron threshold. Between 6 and $7 \mathrm{MeV}$ consistent results are found while below $6 \mathrm{MeV}$ the strong transitions observed in Ref. [15] exceed the average gamma strength in the Oslo data by factors 4 to 5 . However, one should be aware that single transitions are analysed for excitation energies $E_{\mathrm{x}}<7 \mathrm{MeV}$ [15] and the level density of $1^{-}$states excited from the ground state is probably too low to discuss an average behaviour in the PDR region.

\section{Level densities}

Level densities of $1^{-}$states in the excitation energy region from 9 to $12.5 \mathrm{MeV}$ were determined from $\left(p, p^{\prime}\right)$ data [24] using a fluctuation analysis [25]. However, the LDs for ${ }^{208} \mathrm{~Pb}$ derived from the Oslo method represent a different spin window depending on the specific reaction. Thus all results are converted to total level densities using Fermi gas models [8]. This can be achieved using the following equation

$$
\rho^{t o t}(E)=\frac{2 \rho(E, J, \Pi)}{f(J)},
$$

where $\rho^{t o t}(E)$ is the total level density and $\rho(E, J, \Pi)$ is the level density for levels with spin $J$ and parity $\Pi$. The so-called spin distribution function is defined as

$$
f(J)=\frac{2 J+1}{2 \sigma^{2}} \exp \left[-\frac{\left(J+\frac{1}{2}\right)^{2}}{2 \sigma^{2}}\right],
$$

where $\sigma$ is the spin cutoff parameter. Since the spin cutoff depends on the parameters of the Fermi gas model one has to investigate the model dependence. For this purpose, we

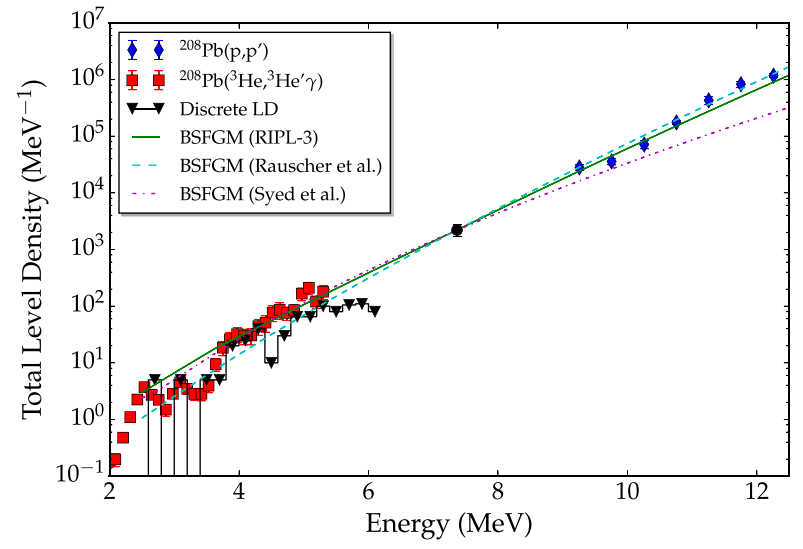

Figure 4. Total LDs from the $\left(p, p^{\prime}\right)$ data $[14,15]$ in comparison to the reanalysed [23] results from the Oslo experiment [22]. The black triangles are results from counting the levels identified in Ref. [28]. The magenta dashed-dotted, green solid and cyan dashed lines are BSFG model predictions with the parameters of Ref. [22], Ref. [13] and Ref. [27], respectively.

considered three parameter sets derived within the backshifted Fermi gas model (BSFG) approach [26]. These include the one used in the original analysis of the Oslo experiment [22], a global set recommended in RIPL-3 [13], and the parametrisation of Ref. [27] developed for $s$-process reaction network calculations, which has been shown to provide a good description of LD for many nuclei near the valley of stability.

The absolute values of the spin distribution function depend on the chosen parametrisation but all three models predict a similar energy dependence in the studied energy region. Therefore, we use averaged values for the comparison with the Oslo data.

The comparison of total level densities from $\left(p, p^{\prime}\right)$ (blue diamonds) with the Oslo results (red squares) is presented in Fig. 4. The value at neutron threshold (black circle) is deduced from $p$-wave resonance neutron capture converted to a total LD with the aid of the RIPL-3 BSFG parametrisation. The black downward triangles denote the results from level counting from a recent study claiming essentially complete spectroscopy up to $6.2 \mathrm{MeV}$ [28].

The magenta dashed-dotted, green solid, and cyan dashed lines are the BSFG model predictions with the parameters of Ref. [13, 22,27]. The models are normalised to the data point at $S_{n}$ with factors $1.15,2.18$ and 0.52 . However, absolute values for the RIPL-3 parametrisation are obtained by normalising to $s$-wave neutron capture resonance spacings. As pointed out in Ref. [22], the data are rather poor in ${ }^{208} \mathrm{~Pb}$ and one should rather normalise to the $p$-wave spacings. The energy dependence of the BFSG model shows differences over the wide energy range spanned by the two data sets. Remarkably, the RIPL-3 parameter set, whose predictions of the total LD are closest to the mean value, provides a consistent description of all data.

\section{Summary and conclusions}

The main aim of this work was to determine the E1, M1 and total GSF of ${ }^{208} \mathrm{~Pb}$ for tests of models recommended in the RIPL-3 data base as well as to study the BA hypothesis by comparison with decay data obtained with the Oslo 
method. It is shown that the E1-GSF can be described well by the SLO and MLO models in the GDR region. In the low-energy region strong fluctuations occur, so that no particular model can be favoured. The average behaviour of the low-energy tail of the IVGDR is best described by the MLO model. However, none of models includes the PDR and thus the predictive power at low excitation energies is generally limited.

The presently recommended parametrisation of the spinflip M1 resonance provides a poor description of the ${ }^{208} \mathrm{~Pb}$ data. Although the absolute magnitude of the resonance maximum is reproduced within a factor of 2 to 3, the width of the M1-GSF is strongly overestimated. As a result the theoretical $B(\mathrm{M} 1)$ strength is - depending on the model - too large by factors 2 to 5 . Since the excitation energy ranges of the spinflip M1 resonance and the PDR overlap in heavy nuclei, this has a strong impact on attempts to extract model parameters for the PDR contribution in decay experiments. Clearly, more data are needed to establish the systematics of the spinflip M1 resonance in heavy nuclei. The method presented in Ref. [21] promises experimental information from the $\left(p, p^{\prime}\right)$ data on spherical $[29,30]$ as well as deformed [31] nuclei.

The comparison of the present GSF derived from ground-state absorption with the Oslo results shows larger values in the PDR energy region. However, the fluctuations of the GSF are very strong due to the anomalously small level densities in the closed-shell nucleus ${ }^{208} \mathrm{~Pb}$, which prevents conclusions on a possible violation of the BA hypothesis in the PDR energy region. Here, tests in openshell nuclei with higher level densities are required and a corresponding study [31] is under way.

Total level densities for ${ }^{208} \mathrm{~Pb}$ were derived from fluctuations of the high-resolution $\left(p, p^{\prime}\right)$ cross sections in the IVGDR energy region and compared to those from the Oslo method covering much lower energies. Using the BSFG model parameters suggested by RIPL-3 to convert the experimental partial-spin results to total level densities, remarkable agreement between the two results is obtained. This provides an independent confirmation of the approach [8] to separate GSFs and LDs in Oslo-type data.

We are indebted to M. Guttormsen and A.C. Larsen for providing us with the revised analysis of the data of Ref. [22]. This work has been supported by the DFG under contract SFB 1245 and by the JSPS, KAKENHI Grant Number JP14740154.

\section{References}

[1] M. Arnould et al., Phys. Rep. 450, 97 (2007)

[2] M.B. Chadwick et al., Nucl. Data Sheets 112, 2887 (2011)

[3] M. Salvatore and G. Palmiotti, Prog. Part. Nucl. Phys. 66, 144 (2011)

[4] M. Wiescher, F. Käppeler and K. Langanke, Annu. Rev. Astron. Astrophys. 50, 165 (2012)

[5] D.M. Brink, Ph.D. thesis, Oxford University (1955)

[6] P. Axel, Phys. Rev. 126, 671 (1962)

[7] P.F. Bortignon, A. Bracco and R. A. Broglia, Giant Resonances: Nuclear Structure at Finite Temperature (Harwood Academic, Amsterdam, 1998)

[8] A. Schiller et al., Nucl. Instrum. Methods Phys. Res., Sect. A 447, 498 (2000)

[9] M. Guttormsen et al., Phys. Rev. Lett. 116, 012502 (2016)

[10] M. Krtička et al., Phys. Rev. Lett. 92, 172501 (2004)

[11] M. Guttormsen et al., Phys. Rev. Lett. 109, 162503 (2012)

[12] J. Enders et al., Phys. Rev. C 71, 014306 (2005)

[13] R. Capote et al., Nucl. Data Sheets 110, 3107 (2009)

[14] A. Tamii et al., Phys. Rev. Lett. 107, 062502 (2011)

[15] I. Poltoratska et al., Phys. Rev. C 85, 041304(R) (2012)

[16] J. Kopecky et al., Phys. Rev. C 47, 312 (1993)

[17] International Atomic Energy Agency, Handbook for Calculations of Nuclear Reaction Data Reference Input Parameter Library (IAEA, Vienna, 1998)

[18] K. Heyde et al., Rev. Mod. Phys. 82, 2365 (2010)

[19] R. Schwengner et al., Phys. Rev. C 81, 054315 (2010)

[20] S. Müller et al., Phys. Rev. Lett. 54, 293 (1985)

[21] J. Birkhan et al., Phys. Rev. C 93, 041302(R) (2016)

[22] N.U.H. Syed et al., Phys. Rev. C 79, 024316 (2009)

[23] M. Guttormsen and A.C. Larsen, private communication (2016)

[24] I. Poltoratska et al., Phys. Rev. C 89, 054322 (2014)

[25] P.G. Hansen et al., Nucl. Phys. A518, 13 (1990)

[26] W. Dilg et al., Nucl. Phys. A217, 269 (1973)

[27] T. Rauscher et al., Phys. Rev. C 56, 1613 (1997)

[28] A. Heusler et al., Phys. Rev. C 93, 054321 (2016)

[29] A.M. Krumbholz et al., Phys. Lett. B 744, 7 (2015)

[30] C. Iwamoto et al., Phys. Rev. Lett. 108, 262501 (2012)

[31] P. von Neumann-Cosel, S. Bassauer and D. Martin, Proceedings Nuclei in the Cosmos XIV, Niigata, Japan (in press); and to be published 